Canadian Journal of Applied Linguistics

Revue canadienne de linguistique appliquée

\title{
Développement de la compétence linguistique permettant l'accord du verbe en nombre à l'écrit chez des élèves de l'élémentaire en contexte minoritaire
}

\author{
Joël Thibeault, Carole Fleuret et Pascale Lefrançois
}

Volume 21, numéro 2, 2018

URI : https://id.erudit.org/iderudit/1057964ar

DOI : https://doi.org/10.7202/1057964ar

Aller au sommaire du numéro

\section{Éditeur(s)}

University of New Brunswick

ISSN

1920-1818 (numérique)

Découvrir la revue

\section{Citer cet article}

Thibeault, J., Fleuret, C. \& Lefrançois, P. (2018). Développement de la compétence linguistique permettant l'accord du verbe en nombre à l'écrit chez des élèves de l'élémentaire en contexte minoritaire. Canadian Journal of Applied Linguistics / Revue canadienne de linguistique appliquée, 21(2), 19-45. https://doi.org/10.7202/1057964ar

\section{Résumé de l'article}

La présente recherche explore le développement de la compétence linguistique qui permet l'accord du verbe en nombre à l'écrit chez huit élèves scolarisés en français dans le sud-ouest de l'Ontario, un milieu francophone minoritaire. Grâce à une conceptualisation qui unit les travaux de chercheurs en langues première et seconde, et à l'analyse des données issues d'une activité de complètement et de productions écrites, nous avons fait émerger trois profils développementaux : le premier groupe de scripteurs fait montre d'une compétence opérationnelle en début d'étude, celle-ci demeurant relativement stable au gré des 13 mois de la recherche. Le deuxième groupe peine à orthographier la marque prototypique de la pluralité verbale au commencement de l'étude et exerce, du début à la fin, un contrôle orthographique lacunaire pour ce qui est de l'objet linguistique retenu. Le dernier groupe, analogue au regroupement précédent en début d'étude, présente un développement rapide au cours de la recherche. Nous discutons les résultats en fonction des recherches menées en langues première et seconde, et mettons l'accent sur les spécificités qui sous-tendent l'apprentissage du français écrit en situation de minorité linguistique.
Copyright (c) Joël Thibeault, Carole Fleuret, Pascale Lefrançois, 2018

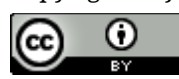

Ce document est protégé par la loi sur le droit d'auteur. L'utilisation des services d’Érudit (y compris la reproduction) est assujettie à sa politique d'utilisation que vous pouvez consulter en ligne.

https://apropos.erudit.org/fr/usagers/politique-dutilisation/ 


\title{
Développement de la compétence linguistique permettant l'accord du verbe en nombre à l'écrit chez des élèves de l'élémentaire en contexte minoritaire
}

\author{
Joël Thibeault \\ Université de Regina \\ Carole Fleuret \\ Université d'Ottawa \\ Pascale Lefrançois \\ Université de Montréal
}

\begin{abstract}
Résumé
La présente recherche explore le développement de la compétence linguistique qui permet l'accord du verbe en nombre à l'écrit chez huit élèves scolarisés en français dans le sud-ouest de l'Ontario, un milieu francophone minoritaire. Grâce à une conceptualisation qui unit les travaux de chercheurs en langues première et seconde, et à l'analyse des données issues d'une activité de complètement et de productions écrites, nous avons fait émerger trois profils développementaux: le premier groupe de scripteurs fait montre d'une compétence opérationnelle en début d'étude, celle-ci demeurant relativement stable au gré des 13 mois de la recherche. Le deuxième groupe peine à orthographier la marque prototypique de la pluralité verbale au commencement de l'étude et exerce, du début à la fin, un contrôle orthographique lacunaire pour ce qui est de l'objet linguistique retenu. Le dernier groupe, analogue au regroupement précédent en début d'étude, présente un développement rapide au cours de la recherche. Nous discutons les résultats en fonction des recherches menées en langues première et seconde, et mettons l'accent sur les spécificités qui sous-tendent l'apprentissage du français écrit en situation de minorité linguistique.
\end{abstract}

\begin{abstract}
This study explores the development of the linguistic competency underlying subject-verb agreement in number in its written form among eight students who were schooled in French in Southwestern Ontario, a francophone minority setting. Through a theoretical framework that unites the work of scholars who focus on first and second language writing development, and the analysis of data collected from a completion task and written productions, three developmental modalities emerged. In the first group, writers developed an operational competency at the beginning of the study, and their competency remained relatively stable throughout the 13 months of the research. The second group of writers showed, throughout this period of time, a competency that does not allow the agreement of verbs in the plural, even when the syntactic environment in which it is written is relatively simple. Finally, the third group demonstrated a competency that was similar to that of the pupils of the second group at the beginning of the study. However, this third group's linguistic competency, between the last two data collection sessions, showed an accelerated development. The data
\end{abstract}


are discussed in light of the results of studies on first and second language writing, and illuminate the specificities related to the learning of written French in minority settings.

\section{Développement de la compétence linguistique permettant l'accord du verbe en nombre à l'écrit chez des élèves de l'élémentaire en contexte minoritaire}

\section{Introduction}

Si, en Ontario, l'anglais est la seule langue qui bénéficie d'un statut sociopolitique officiel, ce sont tout de même quelque 611500 personnes, 4,8 \% de la population, qui s'y déclarent locuteurs du français (gouvernement de l'Ontario, 2016). Pour répondre aux besoins des populations francophones en contexte de minorité linguistique, la Charte canadienne des droits et libertés, en vertu de son article 23, stipule que tout parent ayant luimême fréquenté l'école de langue française a le droit de faire scolariser ses enfants dans un système scolaire de la minorité linguistique officielle (Bélanger, 2015 ; Boutouchent, 2016). Ainsi le ministère de l'Éducation de l'Ontario (MÉO) compte-t-il, de concert avec ses 61 conseils scolaires de langue anglaise, 12 conseils scolaires francophones, qui administrent plus de 450 écoles en province (MÉO, 2018). Située en milieu minoritaire, l'école ontarienne de langue française, en sus d'assurer la fonction traditionnelle de l'éducation, celle de former les citoyens de demain, a également pour mission de contrer l'assimilation graduelle des collectivités francophones minorées et de promouvoir l'ascension sociale des apprenants (Dalley, 2008).

\section{Les élèves scolarisés dans les écoles ontariennes de langue française}

Parce qu'un milieu minoritaire ne peut se définir comme tel que par le côtoiement, sur un territoire donné, de plusieurs langues (Di Meglio, 2014), il convient de reconnaitre, à l'instar de plusieurs chercheurs (Duquette, 2006 ; Gérin-Lajoie, 2012 ; Landry, Deveau et Allard, 2006), que les élèves se socialisant au langage dans ces contextes se construisent inéluctablement une identité composite, au carrefour d'au moins deux langues-cultures. Ces auteurs avancent que les pratiques langagières et culturelles qu'adoptent ces élèves sont souvent, en fonction de différents facteurs, influencées par la langue anglaise, cette dernière étant omniprésente dans leur quotidien. Landry, Allard et Deveau (2010) et Villella (2007), de leur côté, ont noté que plusieurs contextes minoritaires se caractérisent par des taux d'exogamie en croissance et que, de facto, dans ces foyers linguistiquement mixtes, l'utilisation du français se ferait inusuelle, le partenaire anglophone n'ayant que rarement les connaissances linguistiques qui permettent d'en faire une langue de communication familiale. De ce fait, plusieurs enfants arrivent sur les bancs d'école avec un bagage de connaissances restreint en français (Landry, 2010) et, à plusieurs égards, le milieu scolaire doit alors proposer une éducation qui rappelle celle qui est offerte en immersion française (Cormier, 2013).

Dans un autre ordre d'idées, en raison de la mondialisation et des flux migratoires qu'elle favorise, plusieurs chercheurs s'intéressent également à l'inclusion des nombreux élèves issus de l'immigration au sein des écoles de langue française (Farmer, 2016 ; Fleuret, Bangou et Ibrahim, 2013). Afin qu'un nouvel arrivant puisse être scolarisé en français dans la province de l'Ontario, il doit répondre à l'un des deux critères établis par le 
MÉO concernant la scolarisation des immigrants dans ses écoles de langue française : soit le français est la première langue qu'il a apprise et qu'il comprend encore, soit l'un des membres de sa fratrie fréquente aussi une école de langue française au Canada (Immigration Ontario, 2016).

On ne peut donc pas, au regard de l'hétérogénéité des profils linguistiques que nous avons relevée supra, proclamer le monolinguisme des élèves scolarisés au sein des écoles de la minorité francophone, tant s'en faut. Il devient dès lors intéressant de se questionner sur les rôles qui sont joués par les acteurs de la scène éducative vis-à-vis de la pluralité des bagages que portent les apprenants. En effet, l'école de langue française en Ontario a d'abord été conçue dans la foulée de l'État-nation moderne pour préserver la langue et la culture d'une communauté minorisée et définie dans l'homogénéité (Bélanger, 2008) ; en ce moment, elle se redéfinit toutefois en fonction de la diversité ethnolinguistique et ethnoculturelle des élèves qui la fréquentent (Cavanagh, Cammarata et Blain, 2016). Ainsi, face à cette double mission paradoxale de l'école, celle de garantir la pérennité du français en milieu minoritaire et de favoriser l'inclusion des élèves dont les capitaux ne sont pas forcément ceux qui sont reconnus par l'école (Heller, 1999, 2006), le personnel, d'après Bélanger (2007), avancerait l'affirmation sociale d'un usage légitime de la langue en effectuant un travail de négation de la réalité plurilingue des élèves (voir aussi Labrie, 2007). Or, selon Blanchet (2013), cette évacuation du plurilinguisme « empêche les apprenants d'y accéder, [et] considère et maintient en échec les personnes qui développent une véritable compétence plurilingue » (p. 104). On peut donc se demander comment le milieu minoritaire dans lequel ils évoluent et les relations de pouvoir auxquelles ils sont soumis influencent le développement de la compétence linguistique des élèves en français.

\section{L’apprentissage de l'écriture chez les élèves en milieu minoritaire}

Les quelques chercheurs s'étant arrêtés à la description de la compétence linguistique en milieu minoritaire ont surtout centré leurs travaux sur les niveaux postsecondaires. Dans cette optique, les études de Laflamme et Reguigui (2003) et de Bernier (2013) ont montré que, en écriture, le verbe représentait la pierre d'achoppement des étudiants à l'université, ceux-ci peinant à s'engager dans la réflexion grammaticale qui favorise, entre autres, l'accord verbal. Pour ce qui est des ordres préuniversitaires, peu d'études nous permettent de dresser un état des lieux représentatif. En 1995, le groupe DIEPE publiait néanmoins le rapport d'une recherche quantitative internationale dont la visée était la comparaison du savoir-écrire de quatre populations scolaires d'élèves francophones (des Belges, des Français, des Québécois et des Néobrunswickois) qui en sont à leur neuvième année de scolarité obligatoire. Plusieurs faits intéressants ressortent de cette étude : (a) Les notes attribuées à une tâche de production écrite classent les élèves belges en tête (score global moyen : 70,7\%), devant les Québécois (69,6\%) et les Français $(68,7 \%)$. Au dernier rang se trouvent les scripteurs néobrunswickois, avec un score global moyen de 59,4\%. (b) Pour ce qui est est des scores moyens en orthographe lors de cette même production de texte, les élèves du Nouveau-Brunswick sont encore en dernière position, avec un score de 49,3\%. (c) In fine, selon les dires des enseignants néobrunswickois, orthographier d'un point de vue grammatical est une source de difficulté importante pour plus de $50 \%$ des élèves de $9^{\mathrm{e}}$ année.

À ce jour, aucune recherche n'a tenté, à notre connaissance, de comprendre comment les élèves de l'élémentaire en milieu minoritaire opèrent l'accord du verbe à 
l'écrit, bien que cet objet d'apprentissage ait été traité par plusieurs chercheurs en contexte majoritaire (Cogis, 2013 ; Gauvin, 2011 ; Geoffre et Brissaud, 2012). Le domaine du verbe renverrait pourtant à « la zone du plus grand risque orthographique » (Angoujard, 1996, p. 188), comme le montrent les travaux de chercheurs s'étant attachés à décrire la compétence linguistique d'étudiants universitaires en contexte minoritaire (Bernier, 2013 ; Laflamme et Reguigui, 2003).

\section{Le verbe, « un véritable "bastion” orthographique » (Cordary, 2010, p. 78)}

Comme le soulignent Roubaud et Moussu (2010), on ne peut faire l'économie du verbe pour l'apprentissage de l'écrit, car « autour de lui s'organisent les autres éléments (sujet et compléments) » de la phrase (p. 75). Cette catégorie de mot peut toutefois cristalliser d'importantes difficultés chez l'apprenant (Gomila et Ulma, 2014 ; Roubaud et Sautot, 2014), qui doit, pour en faire une utilisation efficiente en production, construire un système de connaissances ayant trait à plusieurs dimensions de la langue (Lepoire-Duq et Ulma, 2010). En effet, parce que le verbe varie en fonction du mode et du temps, il fait intervenir l'environnement énonciatif dans lequel il prend essor. De plus, étant donné que les morphogrammes ${ }^{1}$ indiquant la personne et le nombre du sujet lui sont transmis par un processus d'accord, l'étude du verbe concourt inévitablement à celle de la morphosyntaxe. Enfin, puisque la forme de la base (ou radical) du verbe, c'est-à-dire la partie qui en véhicule le sens, peut connaitre plusieurs variations, il revêt une composante lexicale des plus importantes.

En milieu scolaire, comme le précisent David et Renvoisé (2010), c'est souvent le mode indicatif et le temps présent qui sont initialement privilégiés, en raison de leur soidisant simplicité. Pourtant, le présent de l'indicatif est l'un des paradigmes les plus complexes en français, et ce, pour trois raisons : la distribution des radicaux peut s'y opérer de façon particulièrement irrégulière (p. ex. être en compte cinq, aller en compte quatre), les marques mode-temps y sont pratiquement inexistantes et la majorité des morphogrammes que l'on doit souder aux radicaux à ce mode-temps est inaudible. Pour leur part, Jaffré et David (1999) ajoutent que, là où le nombre, singulier ou pluriel, est conféré aux noms par la situation de communication, le nombre verbal n'est pas sémantiquement motivé ; il est plutôt tributaire de celui du groupe de mots en fonction sujet, ce qui en complique la mise en application (Totereau, Thévenin et Fayol, 1997). Pellat (2009) soutient au demeurant que le nombre du verbe ne peut réellement qu'être étudié à la troisième personne, car la quatrième personne grammaticale, le nous, ne renvoie pas à plusieurs je, alors que la cinquième, le vous, ne renvoie pas à plusieurs $t u$.

Ainsi, à la lumière du panorama socioéducatif que nous avons dressé à l'endroit des milieux francophones minoritaires du Canada et des complexités linguistiques qui soustendent l'utilisation du verbe en production écrite, nous pouvons maintenant arrêter l'objectif de recherche que nous satisferons dans le cadre de cet article. Il s'agira de décrire le développement de la compétence linguistique qui permet l'accord du verbe en nombre à l'écrit au présent de l'indicatif ${ }^{2}$ chez des élèves de la fin de l'ordre élémentaire dans le SudOuest ontarien, la région la plus anglodominante de l'Ontario (Ontario 400, 2016). Pour ce faire, afin de tenir compte de la diversité ethnolinguistique de la population ciblée, nous présentons maintenant la recherche décrivant ladite compétence chez des élèves en langues première (L1) et seconde (L2). D'après Blain (2003), c'est en proposant une telle mise en 
synergie conceptuelle que la recherche pourra, de manière efficiente, prendre en considération l'apprentissage de l'écrit chez des élèves en situation minoritaire.

\section{L’apprentissage de l'accord du verbe en nombre : les études en L1}

En milieu majoritaire, en L1, les chercheurs qui ont d'abord décrit la compétence linguistique sous-tendant l'accord du verbe en nombre en ont proposé une séquentialisation qui correspond à l'âge de l'individu scripteur. Les travaux phares de Totereau (Totereau, Barrouillet et Fayol, 1998 ; Totereau et coll., 1997) ont montré que, en début de scolarité, l'élève n'écrirait que ce qu'il entend et, par conséquent, la morphologie du nombre, fréquemment silencieuse, ne serait pas marquée à l'écrit. Il commencerait ensuite à intérioriser le morphogramme du pluriel nominal, le $s$, lequel se propagerait vers les verbes en $2^{\mathrm{e}}$ année : on assiste dès lors à une surgénéralisation de la marque $s$ qui, pour le scripteur, constitue à ce moment le morphogramme prototypique de la pluralité. Cette surgénéralisation du pluriel des noms aux verbes chuterait toutefois entre la $2^{\mathrm{e}}$ et la $3^{\mathrm{e}}$ année, avant de disparaitre en $4^{\mathrm{e}}$ et en $5^{\mathrm{e}}$ année. Apparaissent parallèlement, en $2^{\mathrm{e}}$ année, les emplois du morphogramme ent pour le verbe. Le recours à ce graphème grimpe ensuite au fil des années, pour être presque complètement maitrisé en $4^{\mathrm{e}}$ année. Ainsi, selon ces travaux, l'élève serait en mesure de repérer le noyau du groupe en fonction sujet et d'accorder le verbe selon ses traits de personne et de nombre en $5^{\mathrm{e}}$ année (voir aussi Guyon, 1997). On peut toutefois, avec Geoffre (2014), reprocher à ces études leur positionnement étapiste, car les recherches récentes qui ont porté sur l'accord du verbe reconnaissent la non-linéarité du parcours menant l'élève à l'expertise orthographique et montrent que, au sortir de l'élémentaire, cette compétence n'est pas complètement construite.

De manière générale, les travaux actuels sur l'accord du verbe en nombre à l'écrit tendent à montrer que, à la fin de l'élémentaire, moment où il doit être appris par les élèves en Ontario français (MÉO, 2006), « la différenciation des marques nominales et verbales, tout comme l'obligation de relier un verbe à son sujet pour déterminer sa finale, composent le noyau dur du savoir des élèves » (Cogis, 2013, p. 81). Toutefois, les chercheurs ayant décrit cet accord à ce moment de la scolarité obligatoire en L1 remarquent tous une grande disparité dans les résultats des élèves (Boyer, 2012 ; Geoffre et Brissaud, 2012). Les surgénéralisations du pluriel nominal seraient marginales (Boyer, 2012 ; Cogis, 2013), et les verbes les mieux réussis seraient ceux qui sont dotés d'une forme supplétive à la troisième personne du pluriel (sont, ont, vont, font), en raison de leur haute fréquence d'occurrence (Boyer, 2012). Enfin, la complexité de la structure phrastique aurait une influence considérable sur la mise en œuvre de l'accord verbal en nombre. Ainsi, si le sujet ne précède pas le verbe, si la liaison entre le donneur et le receveur d'accord est distendue par un rupteur qui est doté d'un nombre grammatical différent de celui du donneur, si le sujet est composé de plusieurs groupes nominaux coordonnés, si le sujet est le pronom relatif invariable qui ou si le donneur d'accord est un nom collectif, les risques d'erreurs liés à l'accord du verbe augmentent. On peut toutefois considérer que certaines de ces erreurs, notamment celles causées par la présence d'un mot-écran devant le verbe, reflètent en fait une expertise orthographique naissante (Gunnarsson-Largy et Largy, 2013), car elles témoignent de l'automatisation progressive des procédures qui concourent à l'accord verbal. En ce sens, l'élève sait que, pour respecter les conventions linguistiques, le verbe doit se transformer en fonction des caractéristiques morphologiques d'un donneur, mais opère son accord selon la proximité syntaxique des mots qui le précèdent. 


\section{L'apprentissage de l'accord du verbe en nombre : les études en L2}

Plus rares, les travaux sur l'accord du verbe en nombre à l'écrit en L2 montrent que le morphogramme prototypique de la pluralité verbale est mis en place relativement tôt, seulement quelques mois après le début de l'apprentissage du français (Granget, 2005 ; Gunnarsson, 2006). À ce moment, les constructions syntaxiques des scripteurs en L2 accuseraient une certaine simplicité, le sujet se situant directement à gauche du verbe et se réalisant le plus souvent sous la forme d'un pronom de conjugaison (Granget, 2005). Cela étant, au fur et à mesure qu'il appréhende les rouages de la L2, le scripteur userait de structures syntaxiques plus élaborées et apprendrait à gérer des environnements phrastiques plus complexes lorsqu'il accorde ses verbes.

La recherche d'Ågren (2008), qui a analysé 400 productions écrites d'apprenants suédophones du français L2 afin de proposer une séquence développementale décrivant l'apprentissage des nombres nominal, adjectival et verbal à l'écrit, précise quant à elle le parcours qui mène le scripteur en L2 à une certaine expertise en écriture. Au début de l'apprentissage, les verbes ne seraient pas fléchis, leur forme infinitive étant souvent privilégiée, mais leur marquage morphologique apparaitrait rapidement, comme d'autres l'ont montré (Granget, 2005 ; Gunnarsson, 2006). Nonobstant une apparition prompte du morphogramme pluriel, une difficulté de taille se manifeste au gré du développement orthographique en L2 : le choix des radicaux conventionnels pour les verbes irréguliers. En effet, les élèves dont le français est la L1 ont été exposés à cette langue depuis leur plus jeune âge et, conséquemment, ont construit un répertoire de connaissances lexicales permettant la sélection des radicaux attendus. De leur côté, les scripteurs en L2, au début de leur parcours, ne peuvent guère prendre appui sur un tel répertoire lors du processus d'accord. De ce fait, quand l'intériorisation du graphème ent commence à se faire remarquer dans les productions écrites en L2, les verbes irréguliers sont fréquemment traités comme des verbes réguliers (p. ex. ils allent). Puis, des instances des verbes sont, ont, font et vont font leur apparition, suivis des autres verbes irréguliers, dès lors marqués conventionnellement sur les plans lexical et morphologique. La chercheuse ajoute que l'accord du verbe peut être soumis à une influence des langues que l'apprenant connait déjà. Ainsi, au début de l'apprentissage du français L2, si les langues de l'élève n'adoptent pas une morphologie verbale qui présente une certaine variation, il est possible que le verbe au présent de l'indicatif, en français, ne soit pas doté de morphogramme grammatical.

En somme, les recherches en L1 et en L2 convergent de plusieurs manières : elles documentent toutes l'appréhension progressive du morphogramme de la pluralité des verbes et l'influence de l'environnement phrastique sur la mise en œuvre du processus d'accord. Les recherches en L2, parce que les scripteurs qui y prennent part doivent conjointement élaborer des connaissances d'ordres morphologique et lexical, montrent que le choix du radical des verbes irréguliers renvoie pour eux à un écueil important. Il devient donc intéressant, en conciliant la recherche en L1 et en L2, de voir comment des élèves de la fin de l'élémentaire en Ontario, un contexte linguistiquement pluriel, développent la compétence linguistique permettant l'accord du verbe en nombre à l'écrit. Dans ce texte, nous répondrons donc à la question suivante : Comment des élèves de la fin de l'élémentaire scolarisés en français dans le Sud-Ouest ontarien développent-ils la compétence permettant d'accorder les verbes en nombre à l'écrit? 


\section{Méthodologie}

Parce que les élèves en contexte minoritaire affichent des connaissances hétérogènes du français (Canavagh et Blain, 2009) et que peu de recherche s'est arrêtée à leur apprentissage de la langue écrite avant l'université (Bernier, 2013), nous avons opté, afin d'opérationnaliser notre étude, pour un devis permettant une analyse axée sur un nombre restreint d'élèves : l'étude de cas (Flyvbjerg, 2011 ; Karsenti et Demers, 2011). Exploratoire, notre étude favorisera une compréhension des changements qualitatifs qui marquent le développement de la compétence liée à l'accord verbal en nombre à l'écrit chez nos participants.

Notre étude de cas est multiple (Stake, 1995), en ce que nous étudions le développement orthographique de plusieurs élèves et que nous comparerons les données provenant de chaque cas afin de mettre au jour des tendances. Gagnon (2012) soutient quant à lui que, pour mener à bien une telle recherche, il est préférable de mobiliser de trois à 12 cas. Ainsi, afin de répondre à notre question de recherche, nous avons sollicité la participation de huit élèves, tous scolarisés en français au sein d'une même école dans le sud-ouest de l'Ontario. Au commencement de l'étude, quatre de ces élèves étaient en $5^{\mathrm{e}}$ année (Ali, Alicia, Emma et Maya), les quatre autres étaient en $6^{\mathrm{e}}$ année (Isaac, Sabrina, Pierre et Kate), et nous les avons suivis pendant 13 mois, de janvier 2015 à février 2016. Il est à noter que, de janvier à juin 2015, les huit élèves étaient dans la même classe multiâges, mais que, de septembre 2015 à février 2016, ils ont été séparés. Ali, Alicia, Emma et Maya étaient alors dans la même classe de $6^{\mathrm{e}}$ année, tandis qu'Isaac, Sabrina, Pierre et Kate faisaient partie d'un même groupe de $7^{\mathrm{e}}$ année. $^{3}$

Tous les participants sont plurilingues et sont scolarisés en français depuis le début de leur scolarité, à l'exception d'Isaac, qui, avant son arrivée au Canada en $5^{\mathrm{e}}$ année, a appris le français L2 à la République d'Ouganda. Pierre a quant à lui été scolarisé dans un programme bilingue français-créole à Haïti ; il est arrivé au Canada en $4^{\mathrm{e}}$ année. Les six autres élèves sont nés en Ontario et y ont toujours fréquenté l'école de langue française. Dans le Tableau 1, nous répertorions les langues que nos participants utilisent à la maison.

Tableau 1

Langue(s) que les participants utilisent lorsqu'ils parlent à leurs parents

\begin{tabular}{lll}
\hline $\begin{array}{l}\text { Élève } \\
\text { (niveau scolaire } \\
\text { en début d'étude) }\end{array}$ & $\begin{array}{l}\text { Langue(s) parlée(s) } \\
\text { avec la mère }\end{array}$ & $\begin{array}{l}\text { Langue(s) parlée(s) } \\
\text { avec le père }\end{array}$ \\
\hline Emma $\left(5^{\mathrm{e}}\right)$ & Anglais & Anglais \\
Maya $\left(5^{\mathrm{e}}\right)$ & Anglais, français & Anglais \\
Ali $\left(5^{\mathrm{e}}\right)$ & Arabe, français, anglais & Anglais, arabe, français \\
Alicia $\left(5^{\mathrm{e}}\right)$ & Anglais, tagalog, français & Anglais \\
Sabrina $\left(6^{\mathrm{e}}\right)$ & Anglais, français, espagnol & Anglais \\
Pierre $\left(6^{\mathrm{e}}\right)$ & Créole haïtien & Créole haïtien \\
Kate $\left(6^{\mathrm{e}}\right)$ & Anglais, français & Anglais \\
Isaac $\left(6^{\mathrm{e}}\right)$ & Swahili, français & Français, anglais \\
\hline
\end{tabular}

Pour décrire le développement de la compétence linguistique liée à l'accord du verbe en nombre à l'écrit, nous avons recouru à trois instruments : l'activité de 
complètement, pendant laquelle le scripteur doit accorder les verbes qui apparaissent à l'infinitif entre parenthèses, la production écrite et l'entretien métagraphique (Jaffré, 1995), lors duquel il doit verbaliser les raisonnements mis en œuvre pendant les tâches précédentes. Dans le cadre de cet article, nous nous focaliserons sur les deux premières tâches.

L'activité de complètement a été utilisée à trois reprises pendant les 13 mois de l'étude : en janvier 2015, en mai 2015 et en février 2016. Pour la concevoir, nous avons d'abord mené une recension des écrits concernant l'influence de l'environnement morphosyntaxique sur l'accord du verbe en nombre à la fin du cours élémentaire en milieu majoritaire (voir notamment Boyer, 2012 et Cogis, 2013) et, pour sélectionner les verbes que les participants devaient accorder, nous nous sommes appuyés sur la typologie de verbes suggérée par Ågren (2008) en L2 : les verbes réguliers en -er, les verbes qui accusent une forme supplétive à la troisième personne du pluriel (sont/ont/vont/font) et les autres verbes. Au total, lors de chaque passation, l'élève devait donc accorder 48 verbes. Qui plus est, tous les verbes retenus font partie de la liste des 100 verbes les plus fréquents en français de Meuleuc et Fauchart (1999), alors que les autres unités lexicales qui sont constitutives de nos items, dans le dictionnaire numérique Antidote (version 8, Druide, 2012), sont dotées de l'indice fréquent ou très fréquent. En contrôlant la fréquence d'occurrence des lexies de nos items, nous voulions que l'attention de l'élève soit portée aux verbes à accorder et qu'il n'ait donc pas à déployer un effort cognitif considérable dans la construction du sens des énoncés. À l'Annexe se trouve un tableau qui expose les environnements syntaxiques retenus pour la construction des items et le nombre de verbes provenant de la nomenclature d'Ågren.

L'analyse des données issues de l'activité de complètement s'est opérée en deux temps. Dans un premier temps, nous avons accordé un point au participant si le morphogramme choisi était morphologiquement plausible (p. ex. il va, ils mangent, ils allent) ; il pouvait dès lors obtenir un score maximal de 48 . Nous avons, dans un deuxième temps, analysé les radicaux des verbes irréguliers. De ce fait, si l'élève avait écrit la base conventionnelle (p. ex. il va), nous lui attribuions un point. Il n'en recevait aucun s'il faisait erreur (p. ex. il alle). Pour cette deuxième analyse, nous avons fait fi des verbes réguliers, lesquels ne varient que peu sur le plan lexical. Le participant était donc susceptible d'obtenir un score maximal de 32.

Pour ce qui est de la production écrite, en janvier 2015 et en février 2016, nous avons demandé aux huit élèves de décrire la première de couverture du livre de jeunesse L'immigration à petits pas (Lamoureux, 2011) et six des images qu'a utilisées Ågren (2008) dans sa recherche, celles-ci mettant en scène deux personnages qui partent pour l'Italie en vacances. La première de couverture de L'immigration à petits pas devait être décrite à l'aide de dix phrases, chaque image tirée d'Ågren devait faire l'objet de trois phrases. C'est donc un minimum de 28 phrases que l'élève devait écrire lors des deux passations.

Pour analyser ces productions écrites, nous avons d'abord repéré les verbes au présent de l'indicatif et nous avons identifié les chaines syntagmatiques dans lesquelles ils s'actualisent (Jaffré et Bessonnat, 1993). Dit autrement, nous avons transcrit, dans un fichier Excel, les verbes et les mots dont ils sont morphosyntaxiquement tributaires. Constituaient donc une chaine syntagmatique le verbe et le groupe de mots en fonction sujet. Parce que les rupteurs, c'est-à-dire les groupes de mots qu'on trouve entre le donneur et le receveur d'accord, peuvent influer sur la réussite des accords (Boyer, 2012 ; Cogis, 
2013), nous les avons également inclus dans ce fichier, et ce, même s'ils ne renvoyaient pas au verbe ou au groupe de mots en fonction sujet.

Dans cet article, nous ferons état des scores généraux de l'activité de complètement et de la production écrite, à toutes les passations. Ensuite, nous nous focaliserons sur trois élèves, Pierre, Maya et Ali, qui affichent des profils de scripteur distincts et qui ont donc obtenu des scores relativement différents les uns des autres. À ce moment, nous reprendrons les scores de l'activité de complètement et nous nous concentrerons sur les constructions syntaxiques des items. Nous verrons donc la performance des trois scripteurs selon la structure phrastique dans laquelle les verbes apparaissent.

\section{Présentation des résultats}

Pour illustrer les résultats de l'activité de complètement, nous avons élaboré des diagrammes à bandes verticales; sur l'axe des abscisses se trouvent les élèves, sur l'axe des ordonnées se trouvent les scores obtenus aux trois passations. Le premier diagramme (Figure 1) présente les résultats liés à la dimension morphologique de l'accord du verbe en nombre, tandis que le deuxième expose ceux liés au choix des radicaux (Figure 2).

Trois modalités semblent se dégager des résultats de nos élèves lors de l'activité de complètement pour ce qui est de la dimension morphologique. Certains élèves, Emma, Pierre et Sabrina, obtiennent des scores élevés dès le début de l'étude. Emma est celle qui, au commencement de la recherche, obtient le score le plus faible des trois (26/48), mais au fil des passations, elle développe sa compétence linguistique et rejoint Sabrina et Pierre $(30 / 48,35 / 48)$. Ce dernier, pour sa part, commence la recherche avec un score de 32/48 et gagne deux points à chaque passation $(34 / 48,36 / 48)$. Quant à Sabrina, en janvier 2015, elle affiche un score de $34 / 48$; elle obtient ensuite $40 / 48$ et 35/48.

Un deuxième regroupement de scripteurs, composé de Maya, de Kate et d'Isaac, commence l'étude avec des résultats plus bas, ces derniers n'évoluant que légèrement de janvier 2015 à février 2016. Maya obtient respectivement, aux trois collectes, 16/48, 14/48 et 20/48. Kate, quant à elle, affiche des résultats similaires, sauf lors de la dernière passation, moment où elle atteint un score modérément à la hausse $(16 / 48,15 / 48,26 / 48)$. Isaac, in fine, affiche des résultats entre $15 / 48$ et 20/48 pendant la recherche $(20 / 48,15 / 48$, 16/48).

Un dernier regroupement de participants, Ali et Alicia, ressemble davantage aux élèves du groupe précédent lors des deux premières passations. Cela étant, entre les deux dernières, ils développent une compétence linguistique qui leur permet d'obtenir des scores analogues à ceux du premier regroupement. Ali, pendant les deux premières collectes, obtient 16/48 et 13/48, alors qu'à la fin de l'étude, son score est de 39/48. Alicia, de son côté, commence l'étude avec $15 / 48$ et $18 / 48$, mais conclut la recherche avec un score de $34 / 48$.

Si on se tourne maintenant vers le marquage lexical des verbes, on note que l'ensemble de nos participants, tout au long de la recherche, fait face à des difficultés. Cependant, l'hétérogénéité des résultats que nous avons constatée à partir de la dimension morphologique de l'accord verbal en nombre à l'écrit ne se manifeste pas de façon aussi évidente en ce qui a trait à son pan lexical. 


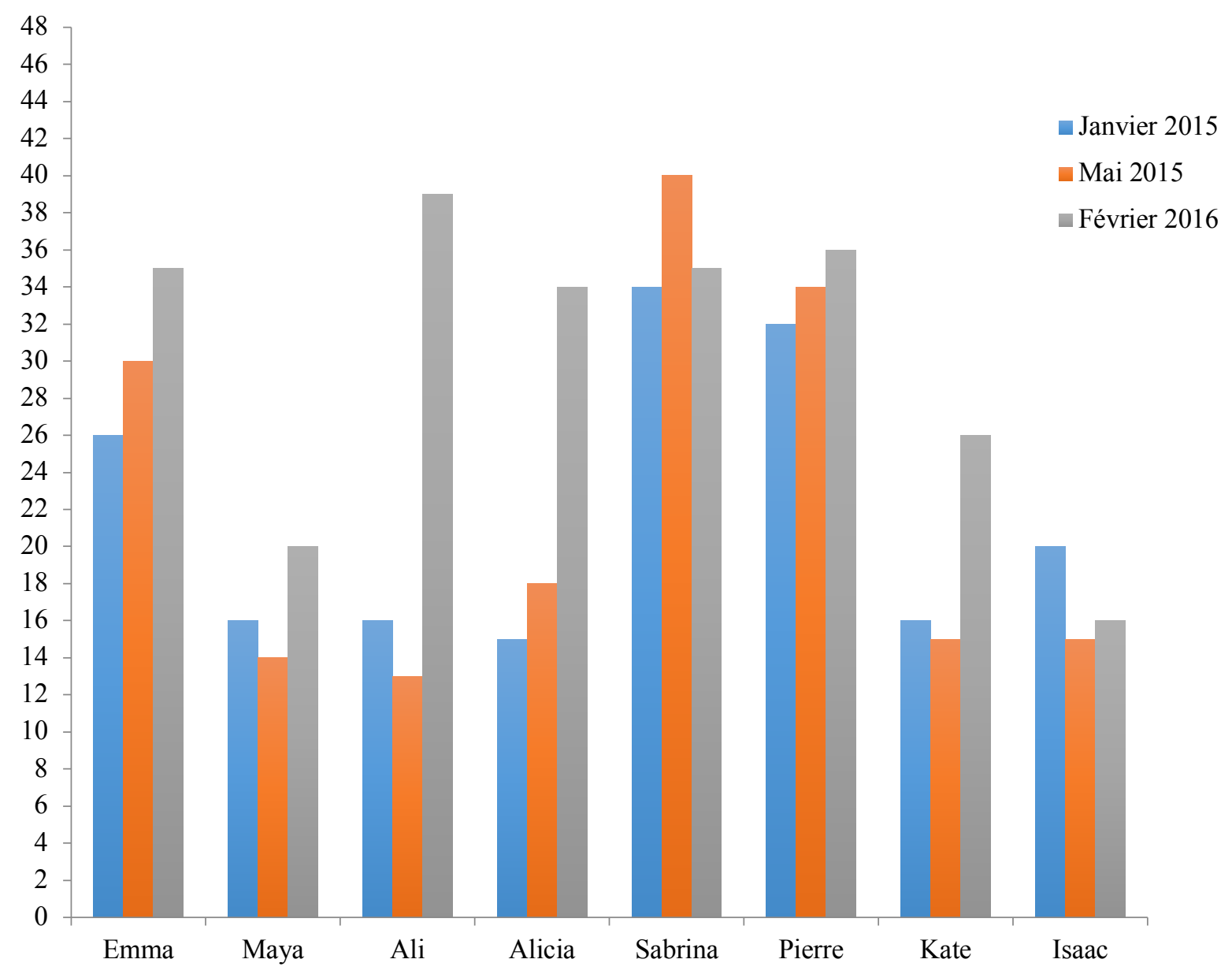

Figure 1. Présentation des scores obtenus pour la dimension morphologique. 


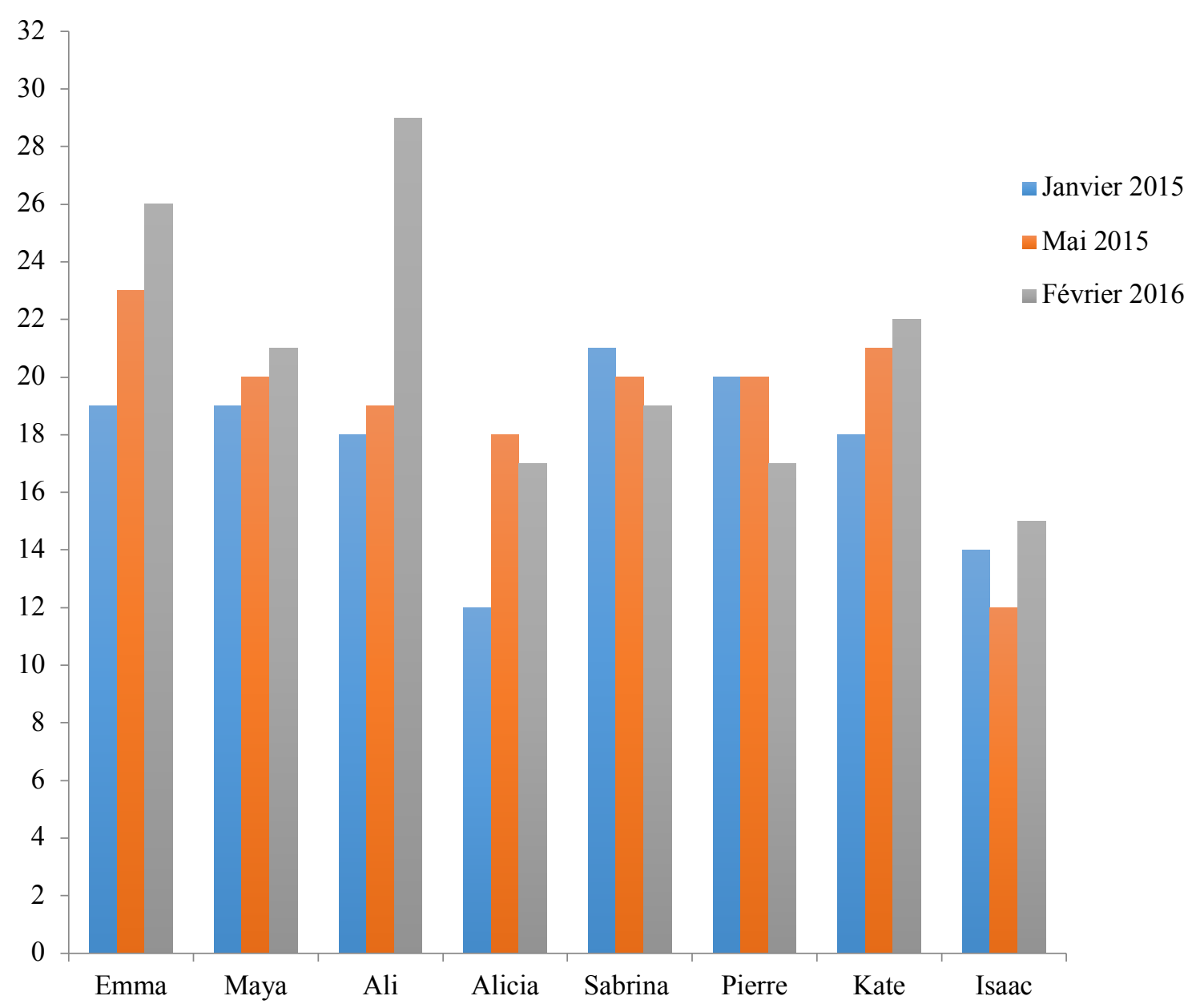

Figure 2. Présentation des scores obtenus pour la dimension lexicale.

Lors des trois collectes, les scores de Kate, de Sabrina, de Maya et de Pierre se situent entre 17/32 et 22/32. Ceux de Kate (18/32, 21/32, 22/32) et de Maya (19/32, 20/32, 21/32) sont faiblement à la hausse au cours de la recherche, tandis que ceux de Sabrina $(21 / 32,20 / 32,19 / 32)$ et de Pierre $(20 / 32,20 / 32,17 / 32)$ diminuent légèrement. Pour leur part, Emma (19/32, 23/32, 26/32) et Ali (18/32, 19/32, 29/32) débutent avec des scores similaires à ceux de leurs quatre camarades, mais enregistrent des progrès plus notables pendant les 13 mois. Alicia, à la première passation (12/32), obtient le score le plus faible du groupe pour l'ensemble de la recherche ; entre la première et la deuxième passation (18/32), elle réalise toutefois un gain important, avant de perdre un point entre la deuxième et la dernière passation (17/32). Le score d'Isaac à la première collecte est plus faible que celui de la grande majorité de ses pairs scripteurs (14/32) et baisse lors de la deuxième $(12 / 32)$. Il réussit néanmoins à gagner quelques points lors de la troisième passation (15/32) ; il demeure quand même celui qui, en fin d'étude, affiche le score le plus bas.

Les données provenant des productions écrites nous permettent de trianguler celles que nous venons de présenter. Cela dit, parce que cette tâche est moins contrôlée que l'activité de complètement, la charge cognitive y étant associée est plus élevée, le scripteur devant respecter des contraintes de différents types en la réalisant (pragmatique, 
syntaxique, orthographique, etc. ; Brissaud, 2015 ; Totereau, Brissaud, Reilhac et Bosse, 2013).

Sur le plan morphologique, les données collectées en production corroborent celles de l'activité de complètement. Nous les présentons en deux tableaux (Tableaux 2 et 3 ), le premier se centrant sur les verbes devant être accordés au singulier, le deuxième s'intéressant aux verbes pluriels. Un troisième tableau (Tableau 4) se focalisera sur les radicaux des verbes.

Tableau 2

Verbes conventionnellement marqués au singulier aux deux passations

\begin{tabular}{lll}
\hline Élève & $\begin{array}{l}\text { Total de verbes bien accordés } \\
\text { en janvier } 2015 \\
(\% \text { de réussite) }\end{array}$ & $\begin{array}{l}\text { Total de verbes bien accordés } \\
\text { en février } 2016 \\
\text { (\% de réussite) }\end{array}$ \\
\hline Emma & $11 / 11(100,0)$ & $11 / 12(91,7)$ \\
Maya & $10 / 12(83,3)$ & $13 / 15(86,7)$ \\
Ali & $10 / 15(66,7)$ & $11 / 11(100,0)$ \\
Alicia & $14 / 17(82,4)$ & $8 / 11(72,7)$ \\
Sabrina & $8 / 9(88,9)$ & $9 / 12(75,0)$ \\
Pierre & $11 / 12(91,7)$ & $8 / 9(88,9)$ \\
Kate & $16 / 17(94,1)$ & $15 / 18(83,3)$ \\
Isaac & $16 / 19(84,2)$ & $16 / 20(80,0)$ \\
\hline
\end{tabular}

Dans l'ensemble, les verbes au singulier ne semblent pas engendrer d'écueils importants chez nos scripteurs. Ali n'a pas su accorder le tiers de ses verbes au singulier $(10 / 25[66,7 \%])$ en début d'étude, mais il les a tous accordés correctement à la fin de la recherche (11/11 [100,0\%]). De manière générale, il appert que l'accord des verbes au singulier, en situation de production écrite, ne pose guère de difficultés particulières aux scripteurs.

Tableau 3

Verbes conventionnellement marqués au pluriel aux deux passations

\begin{tabular}{lll}
\hline & $\begin{array}{l}\text { Total de verbes bien accordés } \\
\text { en janvier } 2015 \\
(\% \text { de réussite })\end{array}$ & $\begin{array}{l}\text { Total de verbes bien accordés } \\
\text { en février } 2016 \\
(\% \text { de réussite) }\end{array}$ \\
\hline Emma & $6 / 12(50,0)$ & $14 / 16(87,5)$ \\
Maya & $2 / 13(15,4)$ & $5 / 15(33,3)$ \\
Ali & $5 / 8(62,5)$ & $15 / 16(93,8)$ \\
Alicia & $1 / 16(6,3)$ & $14 / 15(93,3)$ \\
Sabrina & $12 / 15(80,0)$ & $13 / 13(100,0)$ \\
Pierre & $8 / 13(61,5)$ & $14 / 16(87,5)$ \\
Kate & $4 / 11(36,4)$ & $3 / 10(30,0)$ \\
Isaac & $4 / 10(40,0)$ & $3 / 9(33,3)$ \\
\hline
\end{tabular}

Le portrait des connaissances morphologiques associées à la pluralité des verbes en production écrite témoigne d'une diversité plus importante que celle que nous avons mise en exergue pour les verbes singuliers. Sabrina et Pierre, au commencement de la recherche, 
semblent déjà avoir développé une compétence opérationnelle, mais faillible, car ils affichent des scores élevés (12/15 [80,0\%];8/13 [61,5\%]). À ce même instant, Ali fait aussi montre d'une certaine compétence morphologique (5/8 [62,5\%]), alors que les scores des autres élèves sont plus faibles. Emma a su accorder la moitié des verbes pluriels dans ses textes (6/12 [50,0\%]), Kate et Isaac en ont tous les deux accordé quatre (4/11 [36,4\%], $4 / 10[40,0 \%])$. Maya et Alicia, de leur côté, n'ont presque pas opéré d'accord au pluriel au début de la recherche $(2 / 13[15,4 \%] ; 1 / 16[6,3 \%])$.

En fin d'étude, Pierre (14/16 [87,5\%]) et Sabrina (13/13 [100,0 \%]) continuent d'accorder la plupart de leurs verbes pluriels correctement ; Sabrina ne commet d'ailleurs aucune erreur à cet égard. Ali et Emma semblent en outre parfaire leur compétence et présentent un score plus élevé qu'à la passation précédente, soit 15/16 (93,8 \%) et 14/16 $(87,5 \%)$. C'est toutefois Alicia qui, lors de la dernière passation, fait preuve du progrès le plus marquant. En effet, celle qui, au début de la recherche, a obtenu un score faible (1/16 $[6,3 \%])$ a su dépasser la grande majorité de ses pairs pour l'accord des verbes pluriels au dernier moment de passations, et s'est vu attribuer un score de 14/15 (93,3\%). Pour ce qui est des scores de Kate et d'Isaac, ils sont similaires à ceux qu'ils ont affichés lors de la première collecte $(3 / 10[30,0 \%] ; 3 / 9[33,3 \%])$.

Les scores eu égard aux choix des radicaux appropriés en production écrite sont tous relativement élevés, et nos participants ne semblent pas faire montre de difficultés dans ce domaine. Alicia fait toutefois preuve d'une certaine difficulté en début d'étude (12/24 [50,0 $\%])$, mais à la dernière passation, elle rejoint ses camarades plus forts $(16 / 18[88,9 \%])$. Maya, pour sa part, commet cinq erreurs à la première collecte de données (12/17 [70,6\%]) et quatre à la deuxième (13/17 [76,5\%]). Tous les autres élèves, aux deux passations, ne commettent pas plus de trois erreurs. Il importe toutefois de souligner que, dans la production écrite, qui n'est pas contrôlée, ce sont les participants qui choisissent les verbes qu'ils orthographient. Dans cette optique, tous les élèves ont eu tendance à recourir aux mêmes verbes, des verbes qui sont particulièrement fréquents (surtout des variations du verbe attributif être, en raison du caractère descriptif de la tâche à accomplir). Il est donc fort probable qu'ils aient choisi principalement des verbes dont ils connaissaient les variations lexicales, ce qui a logiquement généré moins d'erreurs.

Tableau 4

Verbes irréguliers conventionnellement marqués lexicalement aux deux passations

\begin{tabular}{lll}
\hline & $\begin{array}{l}\text { Total de radicaux } \\
\text { adéquats en janvier } 2015 \\
\text { Élève }\end{array}$ & $\begin{array}{l}\text { Total de réussite) } \\
\text { adéquats en février } 2016 \\
(\% \text { de réussite) }\end{array}$ \\
\hline Emma & $11 / 12(91,7)$ & $17 / 19(89,5)$ \\
Maya & $12 / 17(70,6)$ & $13 / 17(76,5)$ \\
Ali & $15 / 16(93,8)$ & $18 / 19(94,7)$ \\
Alicia & $12 / 24(50,0)$ & $16 / 18(88,9)$ \\
Sabrina & $11 / 13(84,6)$ & $12 / 13(92,3)$ \\
Pierre & $6 / 8(75,0)$ & $11 / 13(84,6)$ \\
Kate & $13 / 14(92,9)$ & $9 / 10(90,0)$ \\
Isaac & $13 / 13(100,0)$ & $14 / 17(82,4)$ \\
\hline
\end{tabular}

En résumé, les résultats globaux que nous avons présentés jusqu'ici nous amènent à tirer certaines conclusions et à justifier le choix des élèves que nous retenons pour la 
prochaine partie de l'article, qui s'intéressera au développement idiosyncrasique de trois scripteurs. Sur le plan lexical, lors de l'activité de complètement, tous les élèves semblent faire face à certaines difficultés puisque, fréquemment, ils peinent à recourir aux radicaux attendus lors de l'activité de complètement. Les résultats liés au pan morphologique de l'accord du verbe en nombre à l'écrit diffèrent davantage d'un élève à l'autre. Dès le début de l'étude, Sabrina, Pierre et Emma semblent déjà avoir développé une compétence opérationnelle, celle-ci demeurant relativement stable au gré de la recherche. D'autres, Isaac, Maya et Kate, sont plus faibles en début d'étude, et leurs résultats n'évoluent que peu pendant les 13 mois de la recherche. Enfin, Ali et Alicia, en janvier 2015, affichent une compétence linguistique faible sur le plan morphologique ; celle-ci se développe toutefois de façon remarquable entre les deuxième et troisième collectes, à un point tel que leurs résultats sont similaires à ceux de Pierre, de Sabrina et d'Emma. ${ }^{4}$

Pour rendre compte de l'hétérogénéité des connaissances des élèves quant à l'accord verbal en nombre à l'écrit et de la diversité développementale qui semble les caractériser, nous choisissons maintenant de nous arrêter sur le profil de trois élèves, Pierre, Maya et Ali, lesquels se distinguent nettement les uns des autres sur le plan morphologique. ${ }^{5} C^{\prime}$ 'est donc sur cette dimension que nous mettrons l'accent dans la prochaine partie de l'article, qui jette un éclairage sur les résultats de l'activité de complètement en fonction des structures syntaxiques des items.

\section{Présentation du profil de Pierre}

Pierre est un élève qui, dès le début de la recherche, sur le plan morphologique, atteint des scores relativement élevés, autant pour les verbes singuliers que pour les verbes pluriels. Comme le montre le Tableau 5, tout au long de l'étude, il réussit ses accords dans les contextes syntaxiques simples; il ne semble pas éprouver de problèmes à accorder les verbes quand leur donneur d'accord se situe immédiatement à gauche du donneur ou quand le donneur est un nom collectif. Il commet certes quelques erreurs dans ces items au fil de la recherche, ce qui pourrait témoigner d'une certaine fragilité orthographique, mais la majorité des verbes qui apparait dans de tels environnements phrastiques est conventionnellement accordée. Pour Pierre, pendant les 13 mois de la recherche, ce sont les items dans lesquels le donneur ne se situe pas directement à gauche du receveur d'accord qui constituent une source d'erreurs. Presque systématiquement, quand l'ordre phrastique canonique n'est pas respecté et que le sujet se trouve à la fin de la phrase, ou quand devant le verbe se trouve un nom qui n'en est pas le donneur d'accord, la marque morphologique est erronée, l'accord étant opéré avec le nom précédant le receveur. 
Tableau 5

Scores de Pierre en morphologie aux items de l'activité de complètement, selon leur structure morphosyntaxique

\begin{tabular}{llll}
\hline & Passation & Passation & Passation \\
Construction/position du sujet dans l'item & 1 & 2 & 3 \\
\hline Pronom de conjugaison singulier & $6 / 6$ & $4 / 6$ & $6 / 6$ \\
Groupe nominal singulier (court) & $4 / 6$ & $4 / 6$ & $5 / 6$ \\
Pronom de conjugaison pluriel & $6 / 6$ & $6 / 6$ & $6 / 6$ \\
Groupe nominal pluriel (court) & $4 / 6$ & $6 / 6$ & $6 / 6$ \\
Long groupe nominal au pluriel (énumération) & $6 / 6$ & $6 / 6$ & $5 / 6$ \\
$\begin{array}{l}\text { Groupe nominal composé d'un noyau singulier et } \\
\text { d'un groupe prépositionnel avec un nom pluriel }\end{array}$ & $0 / 6$ & $1 / 6$ & $0 / 6$ \\
$\begin{array}{l}\text { Sujet singulier évoquant la pluralité } \\
\begin{array}{l}\text { Sujet en fin de phrase, avec un nombre différent } \\
\text { de celui du noyau du groupe en tête de phrase }\end{array}\end{array}$ & $0 / 3$ & $1 / 3$ & $0 / 3$ \\
$\begin{array}{l}\text { Groupe nominal dans lequel il y a une phrase } \\
\text { subordonnée relative dont le dernier nom a un } \\
\text { nombre qui diffère de celui du donneur }\end{array}$ & $1 / 3$ & $1 / 3$ & $2 / 3$ \\
\hline Total & $32 / 48$ & $34 / 48$ & $36 / 48$ \\
\hline
\end{tabular}

\section{Présentation du profil de Maya}

À la lecture du Tableau 6, on remarque que le profil de Maya est différent de celui de Pierre, que la scriptrice n'a pas encore développé la compétence linguistique permettant, de manière régulière, d'opérer les accords du verbe en nombre quand elle écrit. Lorsque les verbes sont au singulier, on note dans son profil une certaine fragilité orthographique, alors que, quand ils sont pluriels, ses scores chutent.

En effet, au début de la recherche, Maya ne semble pas connaitre la marque prototypique de la pluralité verbale à l'écrit, car les quelques points qu'elle reçoit à cet effet renvoient tous à des verbes pluriels irréguliers de haute fréquence (p. ex. sont), qu'elle a probablement orthographiés en recourant à son bagage de connaissances lexicales. ${ }^{6}$ Elle marque certes le pluriel de certains verbes, mais elle utilise, pour ce faire, le pluriel nominal. Au fur et à mesure de l'étude, cela étant, Maya enregistre un certain progrès, notamment parce qu'elle commence à intérioriser le graphème ent. Ce dernier, lors de la dernière passation, n'est toutefois pas utilisé de manière systématique, loin de là, et il est parfois suivi de la marque du pluriel des noms (p. ex. Elles cherchentes). Là où, au début de la recherche, les verbes pluriels réussis étaient principalement les verbes irréguliers fréquents, en fin de recherche, c'est le manque de constance dans ses choix scripturaux qui 
semble caractériser le profil de Maya. Car, bien qu'elle connaissent le graphème pluriel des verbes, elle n'exerce pas un contrôle orthographique qui en permet systématiquement l'accord en nombre.

Tableau 6

Scores de Maya aux items de l'activité de complètement, selon leur structure morphosyntaxique

\begin{tabular}{llll}
\hline & Passation & Passation & Passation \\
Construction/position du sujet dans l'item & 1 & 2 & 3 \\
\hline Pronom de conjugaison singulier & $3 / 6$ & $4 / 6$ & $4 / 6$ \\
Groupe nominal singulier (court) & $4 / 6$ & $3 / 6$ & $3 / 6$ \\
Pronom de conjugaison pluriel & $1 / 6$ & $1 / 6$ & $1 / 6$ \\
Groupe nominal pluriel (court) & $0 / 6$ & $0 / 6$ & $4 / 6$ \\
Long groupe nominal au pluriel (énumération) & $1 / 6$ & $0 / 6$ & $2 / 6$ \\
$\begin{array}{l}\text { Groupe nominal composé d'un noyau singulier et } \\
\text { d'un groupe prépositionnel avec un nom pluriel }\end{array}$ & $4 / 6$ & $2 / 6$ & $1 / 6$ \\
$\begin{array}{l}\text { Sujet singulier évoquant la pluralité } \\
\begin{array}{l}\text { Sujet en fin de phrase, avec un nombre différent } \\
\text { de celui du noyau du groupe en tête de phrase }\end{array}\end{array}$ & $0 / 3$ & $0 / 3$ & $1 / 3$ \\
$\begin{array}{l}\text { Groupe nominal dans lequel il y a une phrase } \\
\text { subordonné relative dont le dernier nom a un } \\
\text { nombre qui diffère de celui du donneur }\end{array}$ & $1 / 3$ & $1 / 3$ & $2 / 3$ \\
\hline Total & & & \\
\hline
\end{tabular}

\section{Présentation du profil d'Ali}

On note, à partir du Tableau 7, qu'en janvier 2015, le profil d'Ali est comparable à celui de Maya. L'accord des verbes au singulier est opéré de façon disparate ; il choisit parfois le morphogramme attendu, mais il fait erreur d'autres fois. Fait intéressant, aux deux premières passations, on note dans le profil d'Ali certains verbes singuliers qui ne sont pas dotés de morphogramme grammatical (p. ex. Le camion rouge pass). ${ }^{7}$ Les verbes pluriels, de leur côté, ne sont pas marqués du morphogramme approprié. Les quelques verbes pluriels conventionnellement orthographiés sont, comme chez Maya, des verbes irréguliers à haute fréquence. On peut donc croire qu'Ali, lors des deux premières collectes, ne connait pas le graphème prototypique des verbes pluriels, d'autant plus que, quand le donneur est un nom collectif, il surgénéralise parfois le $s$ du pluriel des noms.

Entre la deuxième et la dernière passation, Ali fait toutefois preuve d'un progrès remarquable et se situe, à cet instant de la recherche, parmi les scripteurs les plus 
compétents de notre échantillon. Il opère un contrôle orthographique presque complet lorsqu'il accorde les verbes au singulier et au pluriel. De surcroit, il connait visiblement le graphème de la pluralité verbale, dont il fait fréquemment usage selon les normes en vigueur. Il produit à quelques reprises des accords par proximité et accorde parfois le verbe au pluriel avec un nom collectif. Au demeurant, à l'instar de l'ensemble des participants de notre échantillon, tout au long de la recherche, Ali éprouve de la difficulté quand le donneur d'accord se trouve après le verbe.

Tableau 7

Scores d'Ali aux items de l'activité de complètement, selon leur structure morphosyntaxique

\begin{tabular}{|c|c|c|c|}
\hline Construction/position du sujet dans l'item & $\begin{array}{l}\text { Passation } \\
1\end{array}$ & $\begin{array}{l}\text { Passation } \\
2\end{array}$ & $\begin{array}{l}\text { Passation } \\
3\end{array}$ \\
\hline Pronom de conjugaison singulier & $5 / 6$ & $2 / 6$ & $5 / 6$ \\
\hline Groupe nominal singulier (court) & $3 / 6$ & $4 / 6$ & $5 / 6$ \\
\hline Pronom de conjugaison pluriel & $1 / 6$ & $1 / 6$ & $6 / 6$ \\
\hline Groupe nominal pluriel (court) & $1 / 6$ & $0 / 6$ & $6 / 6$ \\
\hline Long groupe nominal au pluriel (énumération) & $1 / 6$ & $1 / 6$ & $6 / 6$ \\
\hline $\begin{array}{l}\text { Groupe nominal composé d'un noyau singulier et } \\
\text { d'un groupe prépositionnel avec un nom pluriel }\end{array}$ & $2 / 6$ & $3 / 6$ & $4 / 6$ \\
\hline Sujet singulier évoquant la pluralité & $2 / 6$ & $2 / 6$ & $4 / 6$ \\
\hline $\begin{array}{l}\text { Sujet en fin de phrase, avec un nombre différent } \\
\text { de celui du noyau du groupe en tête de phrase }\end{array}$ & $0 / 3$ & $0 / 3$ & $0 / 3$ \\
\hline $\begin{array}{l}\text { Groupe nominal dans lequel il y a une phrase } \\
\text { subordonnée relative dont le dernier nom a un } \\
\text { nombre qui diffère de celui du donneur }\end{array}$ & $1 / 3$ & $0 / 3$ & $3 / 3$ \\
\hline Total & $16 / 48$ & $13 / 48$ & $39 / 48$ \\
\hline
\end{tabular}

\section{Discussion}

Notre discussion du développement de la compétence linguistique qui sous-tend l'accord verbal en nombre à l'écrit chez nos participants se déroulera en deux temps. D'abord, nous en traiterons la dimension lexicale. Puis, nous nous pencherons sur sa dimension morphologique, grâce à laquelle nous avons pu faire émerger des profils de scripteurs.

La description de la connaissance lexicale des formes fléchies du verbe au présent de l'indicatif et de son développement nous a d'abord permis de mettre au jour la pertinence d'une jonction des cadres théoriques en L1 et en L2 pour la recherche 
s'intéressant à l'appréhension du français écrit dans le milieu minoritaire où s'est réalisée cette étude (Blain, 2003). En effet, lors de l'activité de complètement, quoique tous les verbes que nous avons choisis soient relativement fréquents en français, nos huit participants ont éprouvé des difficultés dans la sélection des radicaux attendus, comme la recherche sur 1'écriture en français L2 1'a documenté (Ågren, 2008 ; Gunnarsson, 2006). Pour ce qui est des connaissances morphologiques liées à l'accord des verbes en nombre, il convient de mentionner d'abord que les modèles étapistes qui ont vu le jour en Europe à la fin des années 1990 (Guyon, 1997 ; Totereau et coll., 1997 ; Totereau et coll., 1998) semblent trouver un écho restreint dans le développement de la compétence linguistique que nous avons décrit ici. En effet, ces chercheurs remarquent tous l'intériorisation du morphogramme prototypique de la pluralité des verbes en $4^{\mathrm{e}}$ année. Quant à nous, dans notre échantillon, il n'y a que trois élèves, Sabrina, Pierre et Emma, qui orthographient cette marque en début d'étude. Bien sûr, plusieurs d'entre eux se l'approprient, à des degrés divers, pendant la recherche, mais il demeure que, en janvier 2015, moment où nos participants sont à la fin du cours élémentaire, la plupart d'entre eux n'y recourent jamais. En ce sens, si, en contexte majoritaire, à ce moment de la scolarité obligatoire, la distinction des graphèmes associés aux pluriels nominal et verbal renvoie au noyau dur du savoir des élèves quand ils accordent leurs verbes en nombre à l'écrit (Cogis, 2013), cela ne semble pas nécessairement être le cas chez les élèves de notre étude.

Nos résultats rejoignent néanmoins ceux de chercheurs en milieu majoritaire de différentes manières. Les auteurs qui se sont arrêtés à la compétence linguistique à la fin de l'élémentaire s'entendent sur un point : elle se manifeste fort différemment d'un élève à l'autre (Boyer, 2012 ; Cogis, 2013 ; Geoffre et Brissaud, 2012). Nos analyses nous ont d'ailleurs permis de mettre au jour trois modalités qui orienteraient le développement de la compétence linguistique permettant l'accord verbal en nombre à l'écrit et de nous focaliser sur le profil de trois élèves qui en affiche un développement distinct. Le profil de Pierre, qui fait preuve d'une compétence opérationnelle pendant les 13 mois de l'étude, montre que, à la fin de l'ordre élémentaire, il est possible d'avoir intériorisé le graphème de la pluralité des verbes et d'y recourir en production. Cela dit, ce scripteur commet régulièrement, pendant les trois collectes, des erreurs d'accord par proximité, ce qui nous mène à croire, à l'instar de Gunnarsson-Largy et Largy (2013), que cet écart à la norme représente en fait une expertise en construction. Qui plus est, au commencement de la recherche, il semble avoir développé une compétence plus sure lors de l'activité de complètement, car le contrôle orthographique qu'il opère en production écrite engendre tantôt la graphie attendue, tantôt l'erreur. ${ }^{8} \mathrm{Au}$ fil de l'étude, bien que le développement dont Pierre fait preuve dans l'activité de complètement soit limité, il s'améliore en production écrite, et les quelques erreurs qu'il commettait initialement diminuent. Nous croyons donc que le développement de Pierre, pendant les 13 mois, s'est davantage opéré en ce qui a trait à la gestion des contraintes liées à la production d'un texte. Ce résultat rejoint d'ailleurs les dires de certains (Brissaud, 2015 ; Totereau et coll., 2013), qui postulent que les tâches contrôlées comme l'activité de complètement sont plus faciles à réaliser, car l'élève n'a qu'à fixer son attention sur une composante de la langue à la fois.

Le profil de Maya est fort distinct de celui de Pierre. Si distinct qu'il nous est même difficile de mettre en relation ses résultats avec les écrits scientifiques s'étant intéressés à l'apprentissage de l'accord du verbe chez des élèves d'un âge similaire en contexte majoritaire. En effet, les résultats de Maya nous paraissent nettement inférieurs à ceux qu'ont pu observer d'autres chercheurs en France et au Québec (Boyer, 2012 ; Geoffre et 
Brissaud, 2012). Cette caractéristique, de manière générale, nous pousse d'ailleurs à croire que l'environnement sociolinguistique minoritaire dans lequel elle apprend le français peut influer sur son développement de connaissances liées à la morphologie verbale. Boyer (2012), comme nous, remarque qu'il existe un écart de réussite considérable entre les élèves. Elle explique toutefois cette différence interindividuelle par la complexité de la structure phrastique dans laquelle s'insère le verbe à accorder. Si nous avons pu constater l'importance de l'environnement syntaxique lors de notre analyse des données associées à Pierre, nous ne pouvons guère en dire autant pour ce qui est de Maya, cette dernière, pendant les deux premières collectes, n'orthographiant jamais le morphogramme de la pluralité verbale. Quand elle marque le verbe au pluriel, ce qui est chose rare, elle surgénéralise le morphogramme des noms et des adjectifs pluriels. Cette surgénéralisation, par ailleurs, se fait marginale dans les corpus de Boyer et de Cogis (2013), collectés auprès d'élèves du même âge en milieu majoritaire. En fin d'étude, la marque de la pluralité des verbes fait son apparition, mais n'est guère utilisée conventionnellement de manière fréquente. En fait, elle recourt parfois au $s$ du pluriel nominal, qu'elle orthographie à la suite de la marque du pluriel verbal.

Ali, de son côté, se démarque en raison du développement remarquable dont il fait montre entre la deuxième et la dernière collecte de données. Initialement, en janvier 2015, sa compétence est tout à fait analogue à celle des élèves du groupe de Maya; il peine à accorder la majorité des verbes, surtout ceux aux pluriels, il surgénéralise le pluriel nominal, il n'a pas encore intériorisé le morphogramme ent. De plus, il omet parfois le morphogramme à la fin des verbes. Cette absence de morphogramme est tout à fait intéressante et, pour l'expliquer, nous postulons l'existence d'une influence translinguistique avec l'anglais, une langue dont les verbes ne varient que peu sur le plan morphologique. En L2, Ågren (2008) avance d'ailleurs que la morphologie verbale des langues connues par le scripteur peut influencer la mise en œuvre des morphogrammes en français. En fin d'étude, cela dit, son profil est similaire à celui de Pierre, en ce sens qu'il propose des graphies conventionnelles de manière plus systématique. Il orthographie le morphogramme de la pluralité des verbes quand l'environnement syntaxique l'exige et commet des erreurs qui témoignent d'une expertise en croissance.

\section{Conclusion}

Le présent article, rappelons-le, visait à décrire le développement de la compétence linguistique qui permet l'accord du verbe en nombre à l'écrit chez des élèves de la fin de l'élémentaire dans un milieu particulièrement anglodominant : le sud-ouest de l'Ontario. Quoique, en raison du devis de recherche que nous avons retenu, nous n'oserions pas proclamer la représentativité de nos résultats, nous aimerions proposer des pistes didactiques qui répondraient aux besoins des élèves qui ont pris part à cette étude et qui, peut-être, s'avèreraient pertinentes pour les apprenants en milieu francophone minoritaire.

Pour appuyer ces apprenants dans le développement de connaissances lexicales en lien avec le verbe, il nous parait important de prôner un enseignement des verbes qui repose sur leur fréquence d'occurrence (Roy-Mercier et Chartrand, 2016). Tisset (2005), dans cette perspective, conseille de présenter les verbes aux élèves en fonction du nombre de radicaux pour un mode-temps précis. Pour le présent de l'indicatif, par exemple, on retrouvera donc les verbes dotés d'un seul radical (la plupart des verbes en -er [aim-], offrir [offr-], etc.) ; les verbes dotés de deux radicaux (certains verbes en -er [appel-, appelle-], finir [fini-, 
finiss-], etc.) et les verbes dotés de trois radicaux (vouloir [voul-, veu-, veul-], venir [vien-, ven-, vienn-], etc). Les verbes être, avoir, aller et faire devront toutefois être abordés individuellement, car ils ne s'insèrent pas dans cette typologie. Bien que ces propositions didactiques aient été mises en évidence par des chercheurs en milieu majoritaire, nous croyons qu'elles s'avèrent particulièrement essentielles en contexte minoritaire puisque nos participants, même pour les verbes à haute fréquence, ne choisissent pas toujours les radicaux attendus. Il serait donc important que, dans les prochaines années, des chercheurs en contexte de minorité linguistique s'attachent à mettre en œuvre et à décrire l'impact de ces propositions sur l'apprentissage du verbe chez les élèves.

Du côté morphologique, il semble qu'un enseignement différencié soit de mise, car les élèves ont développé des connaissances distinctives les uns des autres. Il est possible que, pour certains d'entre eux, dont Maya, la conceptualisation de la catégorie du verbe en soit encore à ses balbutiements, ce qui expliquerait notamment la surgénéralisation de la marque associée au pluriel des noms. Avec de tels apprenants, il conviendrait donc de mettre en œuvre des démarches d'enseignement du verbe grâce auxquelles les élèves sont appelés à en découvrir les propriétés syntaxiques, morphologiques et sémantiques (Gourdet, Cogis et Roubaud, 2016). En effet, si la recherche en contexte majoritaire soutient que cette conceptualisation semble bien entamée chez les élèves de la fin de l'élémentaire (Cogis, 2013), cela ne semble pas être le cas pour une grande partie de nos participants du même âge en milieu minoritaire. Ainsi, avant même d'aborder l'accord verbal avec les élèves, l'enseignant en contexte minoritaire gagnerait à s'assurer qu'ils ont développé les connaissances du verbe qui en permettent l'accord. Quand les apprenants ont suffisamment conceptualisé le verbe, l'enseignant peut sélectionner les modes-temps qu'il souhaite aborder en fonction de leurs besoins et débuter par les verbes les plus fréquents. Pour en enseigner l'accord, il commencera par mettre en évidence les liens morphosyntaxiques qui unissent le noyau du groupe en fonction sujet et le verbe en recourant aux pronoms de conjugaison. Ainsi pourra-t-il faire ressortir, de manière évidente, les liens entre le donneur et le receveur d'accord. Puis, il complexifiera progressivement la syntaxe des phrases afin que l'élève puisse s'entrainer à opérer l'accord dans des environnements phrastiques dont la complexité syntaxique fluctue.

En définitive, nos données, parce qu'elles ne trouvent qu'un écho partiel dans les résultats de chercheurs en contexte majoritaire, montrent surtout l'importance, en didactique, d'une prise en considération féconde du milieu socioculturel dans lequel évoluent les apprenants. En ce sens, nous préconisons, pour les milieux francophones minoritaires, la création de ce que Rispail (2005) a nommé une sociodidactique du français, laquelle fait référence à une « (sous)discipline affirmée, (socio)linguistique et didactique mêlées, qui se donnerait pour objet d'étude la vie des langues dans et à travers l'école, dans leurs interactions avec leurs autres usages sociaux » (p. 100). Pour élaborer une telle didactique, sociolinguistes et didacticiens devront toutefois s'unir afin de comprendre les contextes minoritaires et leur influence sur le développement de connaissances en français, d'abord, et concevoir et valider, ensuite, des dispositifs pédagogiques qui trouvent leur ancrage dans la réalité sociolinguistique des élèves.

La correspondance devrait être adressée à Joël Thibeault.

Courriel : joel.thibeault@uregina.ca

Revue canadienne de linguistique appliquée : 21, 2 (2018) : 19-45 


\title{
Notes
}

\begin{abstract}
${ }^{1}$ Rappelons que, selon Catach (1995), l'orthographe du français est un plurisystème composé de trois types de graphèmes. Les morphogrammes, l'un des types de graphèmes, sont des marques qui assurent la transcription écrite de l'information grammaticale (on parle de morphogrammes grammaticaux : le $s$ du pluriel nominal, le ent du pluriel verbal, etc.) et des indices de dérivation (les morphogrammes lexicaux : le $t$ de parent, le $s$ de pris, etc.).
\end{abstract}

${ }^{2}$ Dans la suite de cet article, quand nous nous référerons à l'accord du verbe en nombre, il sera implicite que nous y faisons référence au présent de l'indicatif.

${ }^{3}$ Il s'agit d'un détail important, car il est possible que l'enseignement grammatical différent que les participants ont reçu pendant la deuxième partie de l'étude explique leur développement orthographique. Précisons toutefois que le présent texte mettra l'accent sur l'accord verbal des élèves et qu'il ne se centrera pas sur l'enseignement prodigué. Le lecteur intéressé peut consulter Thibeault (2017).

${ }^{4}$ Bien que nous n'insistions pas sur cet aspect ici, il convient de remarquer qu'Ali et Alicia, entre la deuxième et la troisième collecte, étaient dans la même classe ; par conséquent, il est probable qu'ils aient bénéficié d'un enseignement grammatical qui les a amenés à développer rapidement leur compétence linguistique. Le lecteur intéressé peut consulter Thibeault (2017) pour plus de détails.

${ }^{5}$ Nous choisissons effectivement de focaliser sur la présentation d'élèves qui appartiennent à différents groupes, car nous voulons mettre en évidence l'hétérogénéité des connaissances linguistiques qui est connue en milieu minoritaire (Cavanagh et Blain, 2009). Le lecteur souhaitant savoir comment ces trois élèves se distinguent des membres de leur groupe peut se référer à Thibeault (2017) ; le portrait des huit élèves y est longuement présenté.

${ }^{6}$ Les commentaires métagraphiques qu'émet Maya confirment d'ailleurs cette hypothèse.

${ }^{7} \mathrm{Ce}$ trait n'est pas unique à Ali. On retrouve aussi une telle absence de graphème grammatical dans les écrits d'Isaac et de Kate.

${ }^{8}$ Rappelons que, pour l'accord des verbes pluriels en production écrite, Pierre a obtenu un score de $61,5 \%$ en janvier 2015 et de $87,5 \%$ en février 2016. 


\section{Références}

Ågren, M. (2008). À la recherche de la morphologie silencieuse : sur le développement du pluriel en français L2 écrit (thèse de doctorat inédite). Université de Lund, Suède.

Angoujard, A. (1996). Maitrise des formes verbales : problèmes d'apprentissage, stratégies d'enseignement du CE1 au CM2. Repères, 14(1), 183-199.

Bélanger, N. (2007). Une école, des langues... ? L'enseignement du français en milieu minoritaire en Ontario. Le français aujourd'hui, 158(3), 49-57.

Bélanger, N. (2008). Le lien social à l'épreuve de la modernité tardive : vers une approche compréhensive de l'expérience des enfants face à la diversité à l'école de langue française en Ontario. Éducation et francophonie, 36(2), 98-117.

Bélanger, N. (2015). L'éducation en langue française en Ontario d'hier à aujourd'hui. Problématiser la notion de choix au regard de la forme scolaire. Revue d'histoire de l'éducation, 27(2), 48-63.

Bernier, M. (2013). L'ajout d'erreurs : regard sur un épiphénomène du déficit du savoir grammatical en français au postsecondaire en milieu francophone minoritaire. Éducation francophone en milieu minoritaire, 8(1), 1-17.

Blain, S. (2003). L'enseignement de l'écriture en milieu minoritaire canadien : problématique particulière et complémentarité des cadres théoriques en L1 et L2. Dans J.-M. Defays, J.-L. Delcominette, J.-L. Dumortier et V. Louis (dir.), Langue et communication en classe de français. Convergences didactiques en langue maternelle, langue seconde et langue étrangère (p. 185-200). Cortil-Wodon, Belgique : Éditions Modulaires Européennes.

Blanchet, P. (2013). Standardisation linguistique, glottophobie et prise de pouvoir. Cahiers de linguistique, 39(1), 93-108.

Boutouchent, F. (2016). Le passage du milieu francophone minoritaire au milieu francophone majoritaire : étude d'une expérience d'enseignants en formation pour comprendre l'influence du milieu sur le développement professionnel. Revue canadienne de linguistique appliquée, 19(1), 84-108.

Boyer, P. (2012). La compétence morphographique d'élèves de première secondaire. $L$ 'effet du biais d'évaluation de son efficacité personnelle sur la performance (thèse de doctorat, Université du Québec à Montréal, Canada). Repéré à http://www.archipel.uqam.ca/5352/1/D2405.pdf

Brissaud, C. (2015). Quels enseignements tirer de quatre-vingt-dix-huit dictées de troisième ? Le français aujourd'hui, 190(3), 61-72.

Catach, N. (1995). L'orthographe française ( $3^{\mathrm{e}}$ éd.). Paris, France : Nathan.

Cavanagh, M. et Blain, S. (2009). Relever quatre défis de l'enseignement de l'écrit en milieu francophone minoritaire. Cahiers franco-canadiens de l'Ouest, 21(1-2), 151178.

Cavanagh, M., Cammarata, L. et Blain, S. (2016). Enseigner en milieu francophone minoritaire canadien : synthèse des connaissances sur les défis et leurs implications pour la formation des enseignants. Revue canadienne de l'éducation, 39(4). Repéré à http://journals.sfu.ca/cje/index.php/cje-rce/article/view/2268/1884

Cogis, D. (2013). Du prescrit au réel en CM2 : l'accord sujet-verbe dans le corpus Grenouille. Dans C. Gunnarsson-Largy et E. Auriac-Slusarczyk (dir.), Écriture et réécritures chez les élèves. Un seul corpus, divers genres discursifs et 
méthodologies d'analyse (p. 61-84). Louvain-la-Neuve, Belgique : AcademiaL'Harmattan.

Cordary, N. (2010). L'orthographe du participe passé : les entretiens métagraphiques pour évaluer et comprendre les difficultés des élèves en classe de seconde. Synergies France, 6, 77-84.

Cormier, M. (2013). La francisation dans un contexte de revitalisation langagière. Repéré à https://www.ctf-fce.ca/Publication-Library/FRENQUETES-10-2013-Francisationrevitalisation-langagiere-Resume-fr-01.pdf

Dalley, P. (2008). Principes sociolinguistiques pour l'encadrement pédagogique. Dans P. Dalley et S. Roy (dir.), Francophonie, minorités et pédagogie (p. 281-300). Ottawa, Canada : Presses de l'Université d'Ottawa.

David, J. et Renvoisé, C. (2010). La morphologie verbale : repérer les complexités et les régularités. Synergies France, 6, 61-75.

Di Meglio, A. (2014). Didactiques des langues minoritaires et enjeux de légitimation. Dans P. Blanchet et P. Chardenet (dir.), Guide pour la recherche en didactique des langues et des cultures. Approches contextualisées ( $2^{\mathrm{e}}$ éd., p. 325-333). Paris, France : Éditions des archives contemporaines.

Druide Informatique. (2012). Antidote (Version 8) [Logiciel, CD-ROM]. Montréal, Canada : Druide informatique.

Duquette, G. (2006). Le bilinguisme des élèves inscrits dans les écoles secondaires de langue française de l'Ontario : perceptions, valeurs et comportement langagier. Revue des sciences de l'éducation, 32(3), 665-689.

Farmer, D. (2016). Migrations et « nouvelles mobilités » : regards d'élèves et d'enseignants dans une école de langue française en Ontario. Alterstice, 6(1), 105-119.

Fleuret, C., Bangou, F. et Ibrahim, A. (2013). Langues et enjeux interculturels : une exploration au cœur d'un programme d'appui à l'apprentissage du français de scolarisation pour les nouveaux arrivants. Revue canadienne de l'éducation, 36(4), 280-298.

Flyvbjerg, B. (2011). Case study. Dans N. K. Denzin et Y. S. Lincoln (dir.), The sage handbook of qualitative research (4 éd., p. 301-316). Thousand Oaks, CA : Sage Publications.

Gagnon, Y.-C. (2012). L'étude de cas comme méthode de recherche (2 éd.). Boisbriand, Canada : PUQ.

Gauvin, I. (2011). Interactions didactiques en classe de français :

enseignement/apprentissage de l'accord du verbe en première secondaire (thèse de doctorat, Université de Montréal, Canada). Repéré à

https://papyrus.bib.umontreal.ca/xmlui/bitstream/handle/1866/5281/gauvin_isabelle 2011_these.pdf? sequence $=2 \&$ isAllowed $=\mathrm{y}$

Gérin-Lajoie, D. (2012). L'école et le rapport à l'identité chez les jeunes des minorités des langues officielles. Repéré à http://www.ctf-fce.ca/Publication-

Library/ArticledeDianeGérinLajoie.pdf

Geoffre, T. (2014). Profils d'acquisition de la morphographie au cycle 3. Vers une caractérisation des parcours d'élèves ? Repères, 49, 147-168.

Geoffre, T. et Brissaud, C. (2012). L'accord sujet-verbe : acquis en fin d'école primaire, vraiment? Repéré à http://www.shsconferences.org/articles/shsconf/pdf/2012/01/shsconf_cmlf12_000196.pdf 
Gomila, C. et Ulma, D. (2014). Le verbe en toute complexité : propos introductif. Dans C. Gomila et D. Ulma (dir.), Le verbe en toute complexité. Acquisition, transversalité et apprentissage (p. 9-13). Paris, France : L'Harmattan.

Gourdet, P., Cogis, D. et Roubaud, M.-N. (2016). L'enseignement d'une notion-clé au primaire : le verbe. Dans S.-G. Chartrand (dir.), Mieux enseigner la grammaire. Pistes didactiques et activités pour la classe (p. 147-174). Montréal, Canada : ÉRPI.

Gouvernement de 1'Ontario. (2016). Services gouvernementaux en français. Repéré à https://www.ontario.ca/fr/page/services-gouvernementaux-en-francais

Gouvernement du Canada. (2018). Charte canadienne des droits et libertés. Loi constitutionnelle de 1982. Repéré à https://laws-lois.justice.gc.ca/fra/Const/page15.html

Granget, C. (2005). Développement de l'accord verbal avec un sujet pluriel dans les récits écrits d'apprenants germanophones scolarisés en français. Dans J. Granfeldt et S. Schlyter (dir.), Acquisition et production de la morphologie flexionnelle (p. 111124). Lund, Suède : Perles.

Groupe DIEPE. (1995). Savoir écrire au secondaire. Étude comparative auprès de quatre populations francophones d'Europe et d'Amérique. Bruxelles, Belgique : De Boeck.

Gunnarsson, C. (2006). Fluidité, complexité et morphosyntaxe dans la production écrite en FLE (thèse de doctorat inédite). Université de Lund, Lund, Suède.

Gunnarsson-Largy, C. et Largy, P. (2013). Le développement des automatismes en production écrite du CM2 à la $4^{\mathrm{e}}$ collège à travers l'accord de proximité et les récupérations d'instances. Dans C. Gunnarsson-Largy et E. Auriac-Slusarczyk (dir.), Écriture et réécritures chez les élèves. Un seul corpus, divers genres discursifs et méthodologies d'analyse (p. 129-152). Louvain-la-Neuve, Belgique : Academia-L'Harmattan.

Guyon, O. (1997). Acquisition de l'orthographe du CE1 à la $5^{\mathrm{e}}$ : les morphogrammes grammaticaux $s$ et $n t$. La linguistique : revue de la Société Internationale de Linguistique Fonctionelle, 33(1), 23-40.

Heller, M. (1999). Quel(s) français et pour qui ? Discours et pratiques identitaires en milieu scolaire franco-ontarien. Dans N. Labrie et G. Forlot (dir.), L'enjeu de la langue en Ontario français (p. 129-165). Sudbury, Canada : Prise de Parole.

Heller, M. (2006). Linguistic minorities and modernity (2 éd.). New York, NY : Bloomsbury.

Immigration Ontario. (2016). Éducation en langue française. Repéré à http://www.ontarioimmigration.ca/fr/living/OI_FR_HOW_LIVE_FRENCH_STUD Y.html

Jaffré, J.-P. (1995). Compétence orthographique et acquisition. Dans D. Ducard, R. Honvault et J.-P. Jaffré (dir.), L'orthographe en trois dimensions (p. 94-158). Paris, France : Nathan.

Jaffré, J.-P. et Bessonnat, D. (1993). Accord ou pas d'accord ? Les chaines morphologiques. Pratiques, 77, 25-42.

Jaffré, J.-P. et David, J. (1999). Le nombre : essai d'analyse génétique. Langue française, 124(1), 7-22.

Karsenti, T. et Demers, S. (2011). L'étude de cas. Dans T. Karsenti et L. Savoie-Zajc (dir.), La recherche en éducation. Étapes et approches ( $3^{\mathrm{e}}$ éd., p. 229-252). Saint-Laurent, Canada : ÉRPI. 
Labrie, N. (2007). La recherche sur l'éducation de langue française en milieu minoritaire : pourquoi ? Dans Y. Herry et C. Mougeot (dir.), Recherche en éducation en milieu minoritaire francophone (p. 1-14). Ottawa, Canada : Presses de l'université d'Ottawa.

Laflamme, S. et Reguigui, A. (2003). Homogénéité et distinction. Sudbury, Canada : Prise de parole.

Lamoureux, S. (2011). L'immigration à petits pas. Paris, France : Actes Sud Junior.

Landry, R. (2010). Petite enfance et autonomie culturelle. Repéré à http://www.icrml.ca/images/stories/documents/fr/petite\%20enfancefinal\%20rl_drrl.pdf

Landry, R., Allard, R. et Deveau, K. (2010). École et autonomie culturelle : étude pancanadienne en milieu scolaire francophone minoritaire. Repéré à http://publications.gc.ca/collections/collection_2011/pc-ch/CH3-2-13-2010-fra.pdf

Landry, R., Deveau, K. et Allard, R. (2006). Langue publique et langue privée en milieu ethnolinguistique minoritaire : les relations avec le développement psycholangagier. Francophonies d'Amérique, 22, 167-184.

Lepoire-Duq, S. et Ulma, D. (2010). Enseigner le verbe en français aujourd'hui : enjeux et tensions. Synergies France, 6, 9-15.

Meleuc, S. et Fauchart, N. (1999). Didactique de la conjugaison. Le verbe " autrement». Paris, France : Bertrand-Lacoste.

Ministère de l'Éducation de l'Ontario. (2006). Le curriculum de l'Ontario de la première à la huitième année. Français. Repéré à http://www.edu.gov.on.ca/fre/curriculum/elementary/language18currb.pdf

Ministère de l'Éducation de l'Ontario. (2018). L'éducation en langue française en Ontario. Repéré à http://www.edu.gov.on.ca/fre/amenagement/

Ontario 400. (2016). Statistiques. Repéré à http://ontario400.ca/statistiques/

Pellat, J.-C. (dir.). (2009). Quelle grammaire enseigner? Paris, France : Hatier.

Rispail, M. (2005). Plurilinguisme, pratiques langagières, enseignement : pour une sociodidactique des langues (mémoire d'habilitation à diriger des recherches en sciences du langage). Université de Rennes 2, Rennes, France.

Roubaud, M.-N. et Moussu, M.-J. (2010). Pour une modélisation de l'enseignement de la grammaire au CE1 : l'exemple du verbe. Repères, 41, 71-90.

Roubaud, M.-N. et Sautot, J.-P. (2014). Introduction. Le verbe en friche. Dans M.-N. Roubaud et J.-P. Sautot (dir.), Le verbe en friche. Approches linguistiques et didactiques (p. 11-19). Bruxelles, Belgique : Peter Lang.

Roy-Mercier, S. et Chartrand, S.-G. (2016). L'enseignement du système de la conjugaison pour en favoriser l'apprentissage. Dans S.-G. Chartrand (dir.), Mieux enseigner la grammaire. Pistes didactiques et activités pour la classe (p. 175-200). Montréal, Canada : ÉRPI.

Stake, R. E. (1995). The art of case study research. Thousand Oaks, CA: Sage.

Thibeault, J. (2017). Regard socioconstructiviste sur le développement de la compétence lexicomorphogrammique qui permet l'accord du verbe en nombre chez des élèves de la fin de l'ordre élémentaire dans le Sud-Ouest ontarien (thèse de doctorat, Université d'Ottawa, Canada). Repéré à https://ruor.uottawa.ca/handle/10393/36231?locale $=\mathrm{fr}$

Tisset, C. (2005). Observer, manipuler, enseigner la langue au cycle 3. Paris, France : Hachette. 
Totereau, C., Barrouillet, P. et Fayol, M. (1998). Overgeneralizations of number inflections in the learning of written French: The case of noun and verb. British Journal of Developmental Psychology, 16, 447-464.

Totereau, C., Brissaud, C., Reilhac, C. et Bosse, M. L. (2013). L'orthographe grammaticale au collège : une approche sociodifférenciée. A.N.A.E., 123, 164-171.

Totereau, C., Thévenin, M.-G. et Fayol, M. (1997). Acquisition de la morphologie du nombre à l'écrit en français. Dans L. Rieben, M. Fayol et C. A. Perfetti (dir.), Des orthographes et leur acquisition (p. 147-166). Lausanne, Suisse : Delachaux et Niestlé.

Villella, M. (2007). L'enseignante francophone vivant en situation de mariage exogame (mémoire de maitrise inédit). Université de Toronto, Toronto, Canada. 


\section{Annexe \\ Construction des items de l'activité de complètement}

\begin{tabular}{|c|c|c|c|c|c|}
\hline $\begin{array}{l}\text { Construction/position } \\
\text { du sujet }\end{array}$ & Exemples d'item & $\begin{array}{l}\text { Verbes } \\
\text { en }-e r \\
\text { (nombre } \\
\text { d'items) }\end{array}$ & $\begin{array}{l}\text { Verbes } \\
\text { etre, } \\
\text { avoir, } \\
\text { faire, } \\
\text { aller } \\
\text { (nombre } \\
\text { d'items) }\end{array}$ & $\begin{array}{l}\text { Autres } \\
\text { verbes } \\
\text { (nombre } \\
\text { d'items) }\end{array}$ & Total \\
\hline Pronom personnel singulier & $\begin{array}{l}\text { Il (trouver) beaucoup } \\
\text { d'informations sur les } \\
\text { sites Internet. }\end{array}$ & 2 & 2 & 2 & 6 \\
\hline $\begin{array}{l}\text { Groupe nominal singulier } \\
\text { (court) }\end{array}$ & $\begin{array}{l}\text { Le camion rouge (passer) } \\
\text { près de notre maison tous } \\
\text { les matins. }\end{array}$ & 2 & 2 & 2 & 6 \\
\hline Pronom personnel pluriel & $\begin{array}{l}\text { Ils (dire) toujours la vérité } \\
\text { à leurs parents. }\end{array}$ & 2 & 2 & 2 & 6 \\
\hline $\begin{array}{l}\text { Groupe nominal pluriel } \\
\text { (court) }\end{array}$ & $\begin{array}{l}\text { Ces petits oiseaux (entrer) } \\
\text { dans leur cage. }\end{array}$ & 2 & 2 & 2 & 6 \\
\hline $\begin{array}{l}\text { Long groupe nominal au } \\
\text { pluriel (énumération) }\end{array}$ & $\begin{array}{l}\text { Ma sœur, mes deux frères, } \\
\text { mes parents, ma tante et } \\
\text { mes voisins (rester) } \\
\text { dehors pendant les feux } \\
\text { d'artifice. }\end{array}$ & 2 & 2 & 2 & 6 \\
\hline $\begin{array}{l}\text { Groupe nominal constitué } \\
\text { d'un noyau singulier et d'un } \\
\text { groupe prépositionnel avec } \\
\text { un nom pluriel }\end{array}$ & $\begin{array}{l}\text { Le chat de ses amis (faire) } \\
\text { toujours du bruit en } \\
\text { mangeant. }\end{array}$ & 2 & 2 & 2 & 6 \\
\hline $\begin{array}{l}\text { Sujet singulier évoquant la } \\
\text { pluralité }\end{array}$ & $\begin{array}{l}\text { La population (aller) dans } \\
\text { les écoles pour voter. }\end{array}$ & 2 & 2 & 2 & 6 \\
\hline $\begin{array}{l}\text { Sujet en fin de phrase, avec } \\
\text { un nombre différent de } \\
\text { celui du noyau du groupe } \\
\text { en tête de phrase }\end{array}$ & $\begin{array}{l}\text { Dans le casier (être) les } \\
\text { manteaux. }\end{array}$ & 1 & 1 & 1 & 3 \\
\hline $\begin{array}{l}\text { Groupe nominal dans lequel } \\
\text { il y a une phrase } \\
\text { subordonnée relative dont } \\
\text { le dernier nom a un nombre } \\
\text { différent de celui du } \\
\text { donneur }\end{array}$ & $\begin{array}{l}\text { Le mur que nous décorons } \\
\text { en chantant de belles } \\
\text { chansons (être) très grand. }\end{array}$ & 1 & 1 & 1 & 3 \\
\hline Total & & 16 & 16 & 16 & 48 \\
\hline
\end{tabular}

